\title{
Modeling and control of isochronous and asynchronous traffic flows in a metropolitan area network
}

\author{
R. Bolla, F. Davoli \\ Department of Communications, Computer and Systems Science (DIST) \\ University of Genoa \\ Via Opera Pia 11/A, I-1645 Genova, Italy
}

\begin{abstract}
.
A high-speed network environment is considered, where the multiplexing structure is based on TDM frames, whose slots are dedicated to carrying both isochronous and asynchronous traffic types, generated by a sparse and possibly large user population (e.g., as in a DQDB network). Different classes of isochronous traffic are distinguished, according to their speed and originating user site, and their dynamics is modeled by continuous time Markov chains; the asynchronous packet traffic is represented by a single class. The slots in each frame are dynamically shared among all traffic types, by means of two control levels: a distributed one, acting on the admission of isochronous connection requests independently for each class, and a centralized one, periodically adjusting upper bounds on the number of isochronous slots that may be assigned to each traffic class. The latter assignment is performed by minimizing a cost function related to call blocking and packet delay. The control problem, which is characterized by multiple time scales, is discretized with respect to events occurring in an equivalent overall embedded Markov chain. Several simulation results are reported to test the effectiveness of the method in ensuring Quality of Service requirements.
\end{abstract}

Keywords.

Metropolitan area networks, call admission control, resouce allocation, dynamic hierarchical control.

\section{INTRODUCTION}

Services integration in high speed telecommunication networks aims at offering a common transport to traffic flows characterized by a wide range of different statistical behaviour and performance requirements. In this environment, even in cases where a high 
bandwidth is available, management and control actions play a very important role in guaranteeing a fair allocation of resources (like bandwidth and buffer space) and in ensuring the satisfaction of Quality of Service $(\mathrm{QoS})$ requirements.

To some extent, the specific transfer mode adopted determines the amount and nature of controls, especially with respect to the way bandwidth is allocated within the network (e.g., statistically or deterministically): undoubtedly, Asynchronous Transfer Mode (ATM) networks, where statistical multiplexing provides a very flexible environment at the expense of no guaranteed bandwidth, have received much attention in this context (see, for instance, (Sohraby, 1991)). However, even in the case of networks based on Time Division Multiplexing (TDM) and circuit-switching techniques (possibly integrated with packetswitching in a hybrid solution), the presence of different traffic and service types motivates the problem to decide the sharing of the resources in order to maintain QoS requirements. The bandwidth allocation problem in this context has been treated, for instance, in (Maglaris, 1982), (Kraimeche, 1985), (Viniotis, 1987), (Ross, 1988), (Zukerman, 1989-A), (Zukerman, 1989-B), (Ross, 1989), (Aicardi, 1993), (Bolla, 1993), (Bolla, 1994).

In this paper, we will focus on the case of a Metropolitan Area Network (MAN) based on TDM frames with a hybrid structure, characterized by the presence of two basic traffic types, a circuit-switched one and a packet-switched one. The asynchronous slots in the frame form a common pool, which is accessed by the users by means of a multi-access protocol (as in the DQDB distributed queue algorithm (IEEE, 1990)); moreover, there can be several isochronous traffic classes, which are distinguished according to their speed. A specific isochronous traffic may require one slot every $n$ frames, $n \geq 1$; thus, different isochronous traffic classes effectively "see" frames of different duration. We define a control scheme for the allocation of the resource, which acts on the admission of isochronous call requests and on the assignment of bandwidth to the various users and traffic classes. There are two control levels: i) a fast one acting on the admission of isochronous calls by means of distributed decisional agents (one for each traffic class, residing at the users' Network Interface Units), which operate with the time scale of the call request dynamics; ii) a slower one, playing the role of a coordinator in a hierarchical scheme, which periodically recomputes a set of parameters (related to the bandwidth allocation), by numerically solving a parametric optimization problem, where real time information as well as long term time averages of traffic statistics are taken into account.

The paper will be organized as follows. In the next Section, we first define the frame timing structure and the dynamics of the isochronous and asynchronous traffic. Asynchronous packet arrivals are modeled by a Poisson process, and each packet occupies a time slot in the frame. The isochronous traffic classes are represented by continuous time birth-death processes, corresponding to exponentially distributed interarrival and call duration time intervals. A fixed randomized call admission control rule is then introduced for each isochronous traffic class: at the time of each call request arrival, the rule takes a decision upon its admittance with a certain probability, which is a function of the current number of calls in progress for that class. The form of each function depends on two parameters, and the whole set of parameters is assigned for a fixed time period by the coordinator at the higher control level, which resides at the Network Management Station. In order to define an optimization problem for the coordinator, we consider the embedded Markov chains describing the dynamics of the isochronous traffic, and we define a cost function related to call rejection probability and packet delay. Average values of these quantities are evaluated over the time 


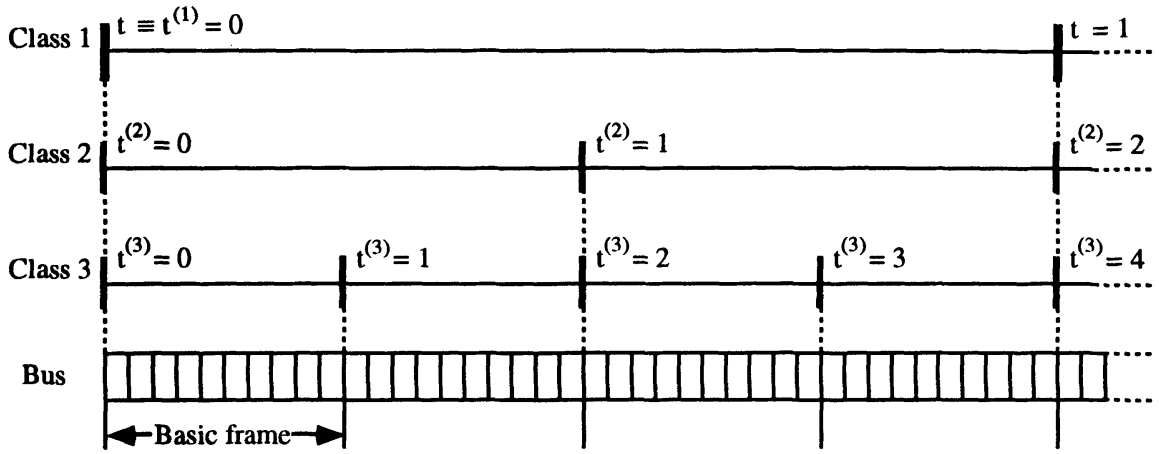

Figure 1. Example of the relation among the time scales for $C=10, H=3, n^{(1)}=4, n^{(2)}=2$ (obviously, $n^{(3)}=1$ ).

horizon corresponding to two successive interventions of the coordinator, and at each intervention new values of the parameters are chosen in order to minimize the cost function, by keeping into account a set of constraints, related to service continuation. The reduction from continuous time processes to a discrete event formulation, the optimization problem and the mathematical programming algorithm used will be described in Section 3. Finally, in Section 4 , several simulation results will be presented that refer to the application of the scheme to a DQDB network, showing its overall behaviour in terms of QoS and dynamic response to variations in the traffic characteristics. Section 5 contains the conclusions.

\section{TIMING STRUCTURE AND CONTROL SYSTEM'S ARCHITECTURE}

We consider a MAN, where $M$ user stations, through their respective Network Interface Units (NIU), see a basic frame of $b$ seconds duration, consisting of a total of $C$ isochronous and asynchronous slots. Each NIU is able to receive isochronous traffic at $H$ different speeds, which will be indicated by $v^{(1)}, \ldots, v^{(H)}$ (in slots/frame), in order of ascending speed. We suppose $v^{(H)}=1$, i.e., the maximum speed achievable by assigning no more than one slot/frame. We also restrict each $v^{(h)}, h<H$, to be of the form $v^{(h)}=1 / n^{(h)}$, where $n^{(h)}$ corresponds to the number of basic frames between two successive slots of a connection at speed $v^{(h)}$. Thus, a slot is always completely filled by the bits corresponding to an isochronous connection; however, a slot needs not be available at every frame for a connection belonging to a speed class that is less than the maximum allowable one. In this respect, an isochronous flow at speed $v^{(h)}, h<H$, effectively "sees" a frame (which we may call a "virtual" frame of speed class $h$ ) that is $n^{(h)}$ times longer than the basic frame. For simplicity reasons that will be apparent in the following, $n^{(1)}$ (corresponding to the lowest speed traffic) is further supposed to be a multiple of all $n^{(h)}, h \neq 1$. Thus, a discrete timing structure is defined and, as a matter of fact, each virtual frame determines a different time scale with respect to the basic frame, characterized by $n^{(h)}$. We assume the longest frame $(h=1)$ as a discrete time reference unit, which will be denoted by $t$. The relationship among the various frames is shown in Figure 1.

Access to the asynchronous slots (that are those unused by the isochronous traffic), is 


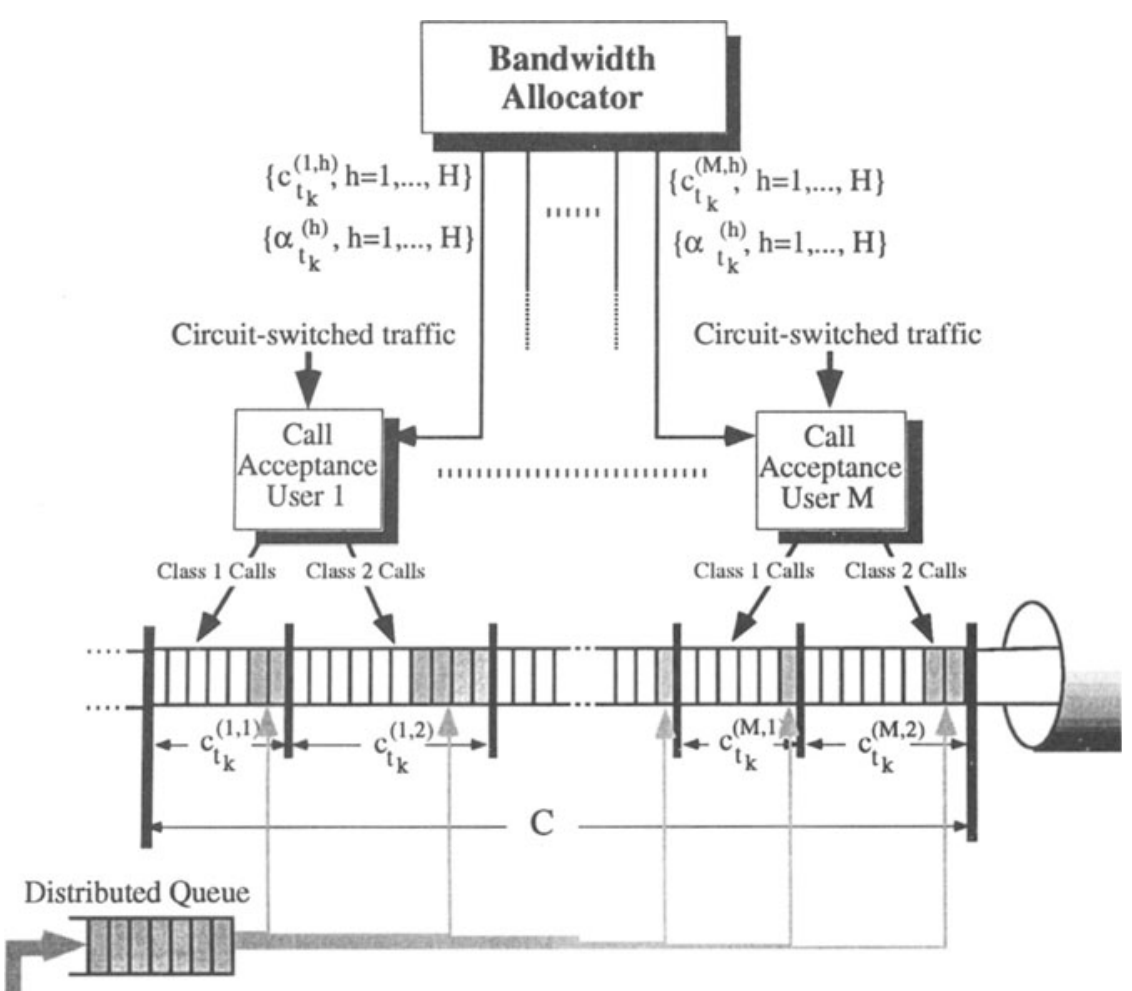

Packet-switched traffic from all the nodes

Figure 2 System structure $(H=2)$.

regulated by a distributed access protocol (e.g., the distributed queue mechanism of DQDB).

Summing up, the basic frame, seen by all users, is essentially divided into two parts, dedicated to carrying isochronous and asynchronous traffic, respectively; the isochronous part is itself divided among the isochronous traffic classes that are distinguished by their originating NIU and their speed. Obviously, these subdivisions are not fixed; we actually want to dynamically control the bandwidth shares, by acting on the acceptance of isochronous calls and on the distribution of isochronous slots among the different classes. The control system's architecture described in the following is depicted in Figure 2.

Let $t_{k}, k=0,1, \ldots$, denote a decision instant, i.e., the frame (in terms of the time variable $t$ as defined above) when each $\mathrm{NIU}_{i}, i=1, \ldots, M$, is assigned capacities $c_{t_{k}}^{(i, h)}, h=1, \ldots, H$, in slots/frame, that can be used for the isochronous traffic. This assignment lasts over the time units $\left[t_{k}, t_{k}+1, \ldots, t_{k+1}-1\right]$, i.e., for the whole time horizon $G \equiv t_{k+1}-t_{k} \equiv T / n^{(1)} b(G$ and $T$ represent the length of the time horizon in terms of the discrete time unit and in seconds, respectively). Any part of this capacity unused by the isochronous traffic can be utilized to 

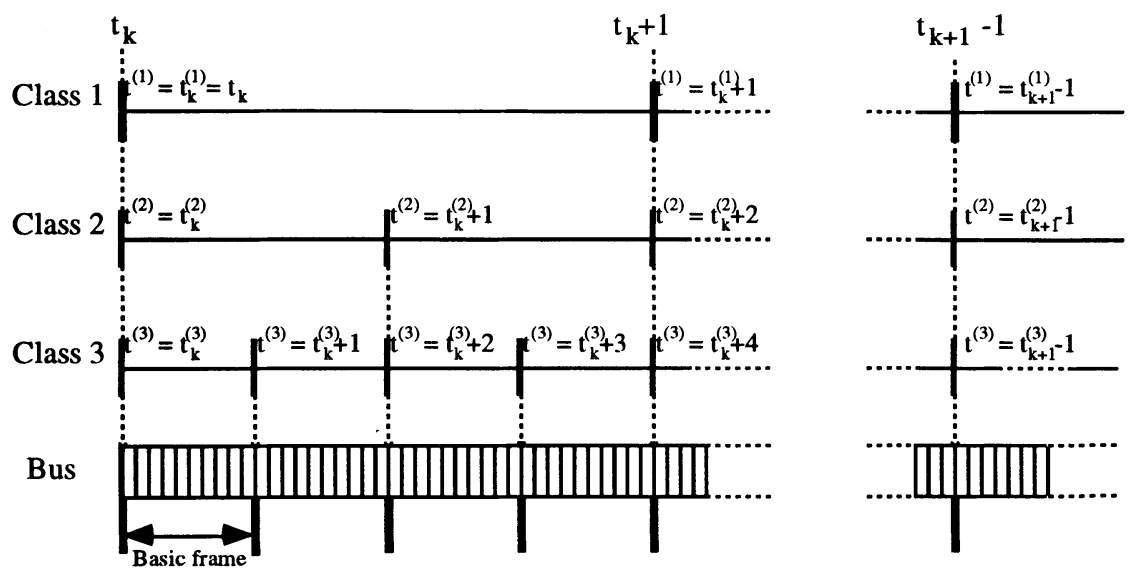

Figure 3 Timing structure and decision instant $t_{k}$.

carry packets of the asynchronous one, which access it according to the multiaccess protocol, and are therefore regarded as a whole undistinguished stream. The timing structure with respect to the decision instants is illustrated in Figure 3.

Variables $c_{t_{k}}^{(i, h)}$ will be considered as continuous; the actual maximum achievable capacity for class $h$ of user $i$ will therefore be $\left\lfloor\tilde{c}_{t_{k}}^{(i, h)}\right\rfloor$, where $\lfloor x\rfloor$ denotes the largest integer less than or equal to $x$, and we have defined the virtual capacity $\tilde{c}_{t_{k}}^{(i, h)}=c_{t_{k}}^{(i, h)} \cdot n^{(h)}$.

We suppose that, in general, each $\mathrm{NIU}_{i}$ may be offered $H+1$ traffic types, namely, $H$ circuit-switched isochronous traffic flows with average call arrival rate $\lambda_{1}^{(i, h)}$ calls/s and average holding time $1 / \mu^{(i, h)} \mathrm{s}$, and a packet switched traffic with average arrival rate $\lambda_{2}^{(i)}$ packets/s, with fixed length packets (one slot). Circuit-switched traffic requires continuation of service until the end of the call.

For the time being, we do not assume any specific distribution for packet arrivals. Actually, the operation of the first control level (made up by the call acceptance controllers) is based solely on the isochronous traffic dynamics and parameters; as regards the higher control level, which is given the responsibility of the capacity allocation, the possible models may differ in the way packet delay is accounted for in the cost function to be minimized. Our choice in this paper is based on an approximation, which does not need to take into account the details of the access algorithm; for its purposes, it suffices to consider asynchronous slots as part of a common pool, and the only information needed regards the overall average packet arrival rate

$$
\lambda_{2} \equiv \sum_{i=1}^{M} \lambda_{2}^{(i)}
$$

Circuit-switched connections of speed class $h$ are supposed to be described by 
continuous-time Markov processes; more specifically, we suppose each isochronous traffic class to be characterized by exponentially distributed and independent interarrival and call holding times, independent of the other classes. We indicate with $\tau$ the continuous time variable. It is worth noting that, in this respect, the present formulation greatly differs from that in (Bolla, 1994), where an entirely discrete time problem was considered: the discretization that will be effected in the next Section is over events, whose time scale is, on the average, much larger than that of frames; this fact will allow to define a more realistic cost function for the higher level controller, extending the optimization horizon for the recomputation of parameters over a range comparable with the average duration of real connections.

Each $\mathrm{NIU}_{\mathrm{i}}$ has a local access controller that acts according to a fixed randomized strategy. More specifically, at the instant $\tau$ of arrival of a connection request of class $h, \mathrm{NIU}_{i}$ either accepts an incoming call (which will be then synchronized with the corresponding frame unit) with probability $p_{\tau}$ or blocks it with probability $1-p_{\tau}$. Let $r_{\tau}^{(i, h)}$ be the number of calls that require continuation of service in the next "virtual" frame (i.e., $n^{(h)}$ basic frames later). As was done in (Aicardi, 1993), (Bolla, 1993) in a different context, we have chosen $p_{\tau}=\beta_{t_{k}}^{(i, h)}\left(r_{\tau}^{(i, h)}\right)$, whose shape is determined by $\tilde{c}_{t_{k}}^{(i, h)}$ and by a further parameter $\alpha_{t_{k}}^{(h)}>0$, as follows

$$
\beta_{t_{k}}^{(i, h)}\left(r_{\tau}^{(i, h)}\right)=\left\{1-\left[\frac{3\left(r_{\tau}^{(i, h)}\right)^{2}}{\left(\tilde{c}_{t_{k}}^{(i, h)}-1\right)^{2}}-\frac{2\left(r_{\tau}^{(i, h)}\right)^{3}}{\left(\tilde{c}_{t_{k}}^{(i, h)}-1\right)^{3}}\right]^{\alpha_{t_{k}}^{(h)}} \quad \begin{array}{l}
\text { if } \tilde{c}_{t_{k}}^{(i, h)}>r_{\tau}^{(i, h)}+1 \\
\text { otherwise }
\end{array}\right.
$$

$$
i=1, \ldots, M ; h=1, \ldots, H ; t_{k} \leq \tau<t_{k+1}
$$

It is not difficult to verify that $\beta_{t_{k}}^{(i, h)}\left(r_{\tau}^{(i, h)}\right) \in[0,1]$ decreases with a reduction of the "available space" $\tilde{c}_{t_{k}}^{(i, h)}-r_{\tau}^{(i, h)}$; moreover, if considered as a function of $\tilde{c}_{t_{k}}^{(i, h)}$, it tends to a step function for $\alpha_{t_{k}}^{(h)} \rightarrow \infty$, whereas it flattens on the horizontal axis for $\alpha_{t_{k}}^{(h)} \rightarrow 0$.

The overall structure of the control system is made up by a two-level hierarchy (see, for instance, (Malinowski, 1989)). The "local" acceptance rules that have been just defined represent a first control level that acts on the basis of local decentralized information; the higher level will be constituted by a central controller, to be described in the next Section, which will act on the parameters $\alpha_{t_{k}}^{(h)}$ and $c_{t_{k}}^{(i, h)}, i=1, \ldots, M, h=1, \ldots, H$. The local controllers have a very simple structure, which has been chosen heuristically. Actually, randomized strategies appear in the literature for some optimal access control problems in the presence of multiple traffic classes (see, e.g., (Maglaris, 1982) and (Ross, 1988)). However, rather than posing a functional optimization problem for the local controllers, which would increase the complexity of the hierarchical scheme, we have preferred to fix the structure of the local strategies a priori, and to optimize only on few parameters. 


\section{SYSTEM'S DYNAMICS AND OPTIMIZATION PROBLEM}

With our choice of $\beta_{t_{k}}^{(i, h)}\left(r_{\tau}^{(i, h)}\right)$, each $r_{\tau}^{(i, h)}, i=1, \ldots, M, h=1, \ldots, H, t_{k} \leq \tau<t_{k+1}$, is a controlled Markov chain with infinitesimal generator $Q_{t_{k}}^{(i, h)}$, whose elements are

$$
q_{j k}^{(i, h)}= \begin{cases}\mu^{(i, h)} j & k=j-1 \\ -\mu^{(i, h)} j-\lambda_{1}^{(i, h)} \beta_{t_{k}}^{(i, h)}(j) & k=j \\ \lambda_{1}^{(i, h)} \beta_{t_{k}}^{(i, h)}(j) & k=j+1 \\ 0 & \text { otherwise }\end{cases}
$$

We define the following cost function for the higher level central controller

$\bar{J}_{t_{k}}=\bar{J}_{t_{k}}^{\text {iso }}+\sigma \bar{J}_{t_{k}}^{\text {asinc }}$

where the first term in the r. h. s. takes into account the performance of the isochronous and the second term that of the asynchronous traffic, and $\sigma$ is a weighting coefficient. We have chosen

$$
\bar{J}_{t_{k}}^{\text {iso }}=\mathrm{E}\left\{\int_{t_{k}}^{t_{k+1}} J_{t_{k}}^{\text {iso }}(\tau) d \tau\right\} \equiv \mathrm{E}\left\{\int_{t_{k}}^{i_{k+1}} \frac{1}{M H} \sum_{i=1}^{M} \sum_{h=1}^{H}\left[1-\beta_{t_{k}}^{(i, h)}\left(r_{\tau}^{(i, h)}\right)\right] d \tau\right\}
$$

where $J_{t_{k}}^{\text {iso }}(\tau)$ represents the call blocking probability, averaged over all users and traffic classes, and we have defined the decision instant in the continuous time unit [seconds] as $t_{k} \equiv t_{k} \cdot n^{(1)} b$.

Let $L_{4}$ be the total number of packets in the distributed packet queue at time $t$; this quantity is obviously part of the overall system's state. However, the description of the dynamics of $L_{t}$, depending on the multiaccess protocol, would be far more complicated than that of the isochronous traffic. With our choice of $\bar{J}_{t_{k}}^{a s i n c}$ below, we can avoid a direct dependence of the cost function on the statistics of $L_{t}$, by making it only a function of the value $L_{t_{k}}$ at time $t_{k}$.

More specifically, we consider the following formulation for $\bar{J}_{t_{k}}^{a s i n c}$, namely

$$
\begin{gathered}
\bar{J}_{t_{k}}^{\text {asinc }}=\max ^{2}\left[0 ; 1-E\left\{\int_{i_{k}^{\prime}}^{t_{k+1}^{\prime}} J_{t_{k}}^{\text {asinc }}(\tau) d \tau\right\}\right] \equiv \\
\equiv \max ^{2}\left[0 ; 1-\frac{\left.\mathrm{E}\left\{\int_{t_{k}^{\prime}}^{t_{k+1}^{\prime}} \sum_{i=1}^{M} \sum_{h=1}^{H} \frac{1}{b \cdot n^{(h)}}\left(\tilde{c}_{t_{k}}^{(i, h)}-r_{\tau}^{(i, h)}\right) d \tau\right\}\right]}{L_{t_{k}}+\lambda_{2} T}\right]
\end{gathered}
$$


The fraction inside the maximum in (6) represents the ratio between the average "available space" for packets over the interval $T=\dot{t}_{k+1}-\dot{t}_{k}$ and the average number of incoming packets over the same interval (including the packet backlog $L_{t_{k}}$ at time $t_{k}$ ).

Considered at the transition instants $t_{e}, e=0,1, \ldots$, each state variable $r_{\tau}^{(i, h)}$ can be described by a Markov chain with transition probabilities

$$
p_{j k}^{(i, h)} \equiv \operatorname{Pr}\left\{r_{t_{e+1}^{(i, h)}}=k \mid r_{t_{e}}^{(i, h)}=j\right\}= \begin{cases}\frac{j \mu^{(i, h)}}{R} & k=j-1 \\ 1-\frac{\lambda_{1}^{(i, h)} \beta_{t_{e}}^{(i, h)}(j)+j \mu^{(i, h)}}{R} & k=j \\ \frac{\lambda_{1}^{(i, h)} \beta_{t_{e}}^{(i, h)}(j)}{R} & k=j+1 \\ 0 & \text { otherwise }\end{cases}
$$

The transition rate $R$ is computed by considering the potential transitions, that is

$$
R=\sum_{i=1}^{M} \sum_{h=1}^{H} \operatorname{Sup}_{s}\left[\mu^{(i, h)} s+\lambda_{1}^{(i, h)} \beta_{t_{k}}^{(i, k)}(s)\right]=\sum_{i=1}^{M} \sum_{h=1}^{H}\left(\mu^{(i, h)} C+\lambda_{1}^{(i, h)}\right)
$$

The equivalence between the model obtained using the actual transition rate of each Markov chain, which is the argument of $\operatorname{Sup}[\cdot]$ in (8), and that obtained using a rate $R$ defined as above is shown, for example, in (Serfozo, 1979); applications of this procedure are found in (Viniotis, 1987), (Rosberg, 1982), (Hajek, 1984). We use the maximum rate with respect to the entire system and not with respect to a single state variable, as we want to describe the evolution of each state variable separately at the same instant $t_{e}$. In principle, we could consider the transition matrix of the whole system; in this case, the actual maximum transition rate would be given by $R$, and the terms in the matrix would describe the transition probability from every possible state variables configuration in $t_{e}$ to every possible configuration in $t_{e+1}$. However, since the variables $r_{\tau}^{(i, h)}, i=1, \ldots, M, h=1, \ldots, H$, are independent of one another, it can be easily seen that the evolution of each single state variable, which could be derived from the complete system matrix, is just that described by (7). For this reason, using the rate $R$, we can consider each Markov chain $r_{\tau}^{(i, h)}$ separately, but we can suppose every chain evolving at the same time instants $t_{e}, e=0,1, \ldots$.

By conditioning the expectations in (5), (6) to the number $W$ of transitions that occurred in $\left[\dot{t}_{k}, \dot{t}_{k+1}\right]$, we obtain 


$$
\begin{aligned}
& \bar{J}_{t_{k}}\left(X_{t_{k}}\right)=\mathrm{E}_{W}\left\{\mathrm{E}_{r, t_{e}}\left\{\sum_{e=0}^{W} \int_{t_{e}}^{t_{e+1}} J_{t_{k}}^{\mathrm{iso}}(\tau) \mathrm{d} \tau \mid W\right\}\right\}+ \\
& +\sigma \max ^{2}\left[0 ; 1-\mathrm{E}_{W}\left\{\mathrm{E}_{r, t_{e}}\left\{\sum_{e=0}^{W} \int_{t_{e}}^{t_{e+1}} J_{t_{k}}^{\mathrm{asinc}}(\tau) \mathrm{d} \tau \mid W\right\}\right\}\right]= \\
& =\mathrm{E}_{W}\left\{\mathrm{E}_{r}\left\{\sum_{e=0}^{W} J_{t_{k}}^{\mathrm{iso}}\left(t_{e}\right) \mathrm{E}\left\{t_{e+1}-t_{e} \mid W\right\}\right\}\right\}+ \\
& +\sigma \max ^{2}\left[0 ; 1-\mathrm{E}_{W}\left\{\mathrm{E}_{r}\left\{\sum_{e=0}^{W} J_{t_{k}}^{\text {asinc }}\left(t_{e}\right) \mathrm{E}\left\{t_{e+1}-t_{e} \mid W\right\}\right\}\right\}\right]
\end{aligned}
$$

where the notation $\mathrm{E}_{r}$ denotes the expectations with respect to $r_{\tau}^{(i, h)}, i=1,2, \ldots, M, h=1,2, \ldots$, $H$, and we have defined $t_{0} \equiv \dot{t}_{k}, t_{W+1} \equiv \dot{t}_{k+1}$. We have explicitly indicated the dependence of $\bar{J}_{t_{k}}$ on $X_{t_{k}} \equiv \operatorname{col}\left[r_{t_{k}}^{(i, h)}, i=1, \ldots, M, h=1, \ldots, H ; L_{t_{k}}\right]$. Expression (9) can be viewed as the cost over a time horizon $T$ for the discrete time decision process, with transition probabilities given by (7). The terms $\mathrm{E}\left\{t_{e+1}-t_{e} \mid W\right\}$ can be computed from the joint density of the $W$ ordered arrival epochs $t_{1} \leq t_{2} \leq \ldots \leq t_{W}$ in the interval $\left[t_{0}, t_{W+1}\right]$ of length $T$, which is given by the $W$ order statistics $W ! / T^{W}$ (see (Wolff, 1987, p. 73)). It turns out that

$$
\mathrm{E}\left\{t_{e+1}-t_{e} \mid W\right\}=\frac{T}{W+1}, \quad e=0,1, \ldots, W
$$

The number $W$ of transitions in the interval $T$ follows an independent Poisson distribution with rate $R$; let

$$
\mathrm{P}(W)=\frac{(R T)^{W}}{W !} e^{-R T}
$$

Moreover, let

$$
\pi_{t_{e}}^{(i, h)}(s)=\operatorname{Pr}\left\{r_{t_{e}}^{(i, h)}=s\right\}
$$

and observe that $0 \leq r_{t_{e}}^{(i, h)} \leq\left\lfloor\tilde{c}_{t_{k}}^{(i, h)}\right\rfloor, \forall t_{e} \in\left[\dot{t}_{k}, i_{k+1}\right)$. Then, by taking into account (10), (11) and (12), we can finally write the expression of the cost function (9) as

$$
J_{t_{k}}\left(X_{t_{k}}\right)=\sum_{W=0}^{\infty}\left\{\sum_{e=0}^{W}\left\{\frac{T}{(W+1)} \bar{J}_{e}^{\text {iso }}\right\} \mathrm{P}(W)\right\}+\sigma \cdot \max ^{2}\left[0 ; 1-\frac{\sum_{W=0}^{\infty}\left\{\sum_{e=0}^{W}\left[\frac{T}{(W+I)} \bar{J}_{e}^{\text {asinc }}\right] \mathrm{P}(W)\right\}}{L_{t_{k}}+\lambda_{2} T}\right]
$$


where we have defined

$$
\begin{aligned}
& \bar{J}_{e}^{\text {iso }}=\frac{1}{M \cdot H} \sum_{i=1}^{M} \sum_{h=1}^{H} \sum_{s=0}^{\left\lfloor\bar{t}_{t_{k}}^{(i, h)}\right\rfloor}\left(1-\beta_{t_{k}}^{(i, h)}(s)\right) \pi_{t_{e}}^{(i, h)}(s) \\
& \bar{J}_{e}^{\text {asinc }}=\sum_{i=1}^{M} \sum_{h=1}^{H} \sum_{s=0}^{\left\lfloor\bar{c}_{t_{k}}^{(i, h)}\right\rfloor} \frac{1}{b \cdot n^{(h)}}\left(\tilde{c}_{t_{k}}^{(i, h)}-s\right) \pi_{t_{e}}^{(i, h)}(s)
\end{aligned}
$$

At each instant $t_{k}, k=0,1, \ldots$, the central agent, basing on the knowledge of all "a priori" information (constituted by $\lambda_{1}^{(i, h)}, i=1, \ldots, M, h=1, \ldots, H$, and $\lambda_{2}$ ), and of $X_{t_{k}}$, wants to assign the maximum capacities $c_{t_{k}}^{(1,1)}, \ldots, c_{t_{k}}^{(1, H)}, \ldots, c_{t_{k}}^{(M, 1)}, \ldots, c_{t_{k}}^{(M, H)}$ and the coefficients $\alpha_{t_{k}}^{(1)}, \ldots, \alpha_{t_{k}}^{(H)}$, in order to minimize (13). In a realizzation of the control system, it is cleary impossible to reach the infinte limits of the sums with the index $W$ in (13); however the summations could been stopped when the value of $\mathrm{P}(W)$ becomes less than a certain threshold (as has been done in the simulations presented in Section 4).

In the capacity assignment the following constraints must be taken into account:

$$
\begin{array}{ll}
\sum_{i=1}^{M} \sum_{h=1}^{H} c_{t_{k}}^{(i, h)}=C, & k=0,1, \ldots \\
\tilde{c}_{t_{k}}^{(i, h)} \geq r_{t_{k}}^{(i, h)}, & i=1, \ldots, M \\
& h=1, \ldots, H \\
k & =0,1, \ldots
\end{array}
$$

where (17) imposes continuation of the outstanding calls. Moreover, as the minimization has to be extended also over the $\alpha_{t_{k}}^{(h)}$ 's, we must consider the additional constraints

$$
\begin{array}{ll}
\alpha_{t_{k}}^{(h)}>0, & h=1, \ldots, H \\
k & =0,1, \ldots
\end{array}
$$

We may say that the $c_{t_{k}}^{(i, h)}$ 's set the partition of the total capacity $C$ among the users and speed classes in relation to the isochronous traffic, and the $\alpha_{t_{k}}^{(h)}$ 's tend to influence the subdivision of the whole available space between the asynchronous and the isochronous traffic.

It is worth noting that, at each intervention time, the actual numbers of active calls $r_{t_{k}}^{(i, h)}$, $i=1, \ldots, M, h=1, \ldots, H$, and the packet backlog in the distributed queue $L_{t_{k}}$ should be known by the centralized decision maker: the first quantities are needed both to correctly initialize the Markov chains and to write constraints (17), whereas $L_{t_{k}}$ explicitly appears in the cost function and creates a feedback effect. Actually, the values of $r_{t_{k}}^{(i, h)}, i=1, \ldots, M, h=1, \ldots, H$, should not create a problem, as calls may be thought of as being assigned by a management function that resides in the same head station as the central controller. On the other hand, $L_{t_{k}}$ 
might be known, with a certain delay, by direct communication on the part of each $\mathrm{NIU}_{i}$ by means of dedicated signaling slots, or might be estimated, with the estimate being used in lieu of the unknown true value.

A final remark must be made with regard to the knowledge of values of the average intensities $\lambda_{1}^{(i, h)}, i=1, \ldots, M, h=1, \ldots, H$, and $\lambda_{2}$, which is also required to evaluate (13). Actually, local estimates can be constructed at $\mathrm{NIU}_{i}$ of $\lambda_{1}^{(i . h)}, \lambda_{2}$, by observing the arrival processes over a time window. These local estimates, in turn, can be communicated to the Network Manager and updated every time that the difference between the old and the new measured values falls above a certain threshold.

Finally, as regards the optimization problem, once computed the gradient, the optimization can be carried out by applying, for example, the gradient projection technique in the same way as in (Aicardi, 1993), with the only difference of having added a new set of optimization variables, namely, $\alpha_{t_{k}}^{(h)}, h=1, \ldots, H$. In fact, the single equality constraint (16) and the simple form of the inequality constraints (17) give the opportunity to obtain very simple gradient projections, that closely match the procedure used in (Bertsekas, 1987) for optimal routing.

\section{SIMULATION RESULTS}

We report and comment in the following several simulation results, whose purpose is to evaluate the effectiveness of the proposed management and control scheme. To fix ideas, we refer to a DQDB network. The simulation is a discrete event one and represents a DQDB network according to the standard (IEEE, 1990).

\section{Network model}

All results have been obtained with a network made up of five user stations and three different classes of isochronous traffic for each user station. Since the DQDB network in a real life situation presents delays between user stations and the access protocol may present unfairness as regards asynchronous traffic, a bandwidth balance technique (BWB) has been utilized.

Now, let us specify the characteristics of the network in full details. Only one bus is considered; the situation is symmetric for the reverse bus. It is important to remember that one of the five NIU's, which corresponds to the head station of the reverse bus, has no traffic on the bus in question and is not counted in $M$.

The frame duration has been set to a standard value of $125 \mu$ s. Given a total capacity of $600 \mathrm{Mbit} / \mathrm{s}$ and a number of bits per slot of 424 (53 bytes), 177 slots/frame are obtained. These slots will be equally subdivided among the different classes of every user at every decision instant in accordance with their isochronous traffic. After fixing a decision interval duration of $2.2 \mathrm{~s}$, the number of frames between two consecutive decision instants results to be 16,160 . Each simulation whose results are illustrated below has a duration of $70.2 \mathrm{~s}$, so as to get 33 decision intervals. 


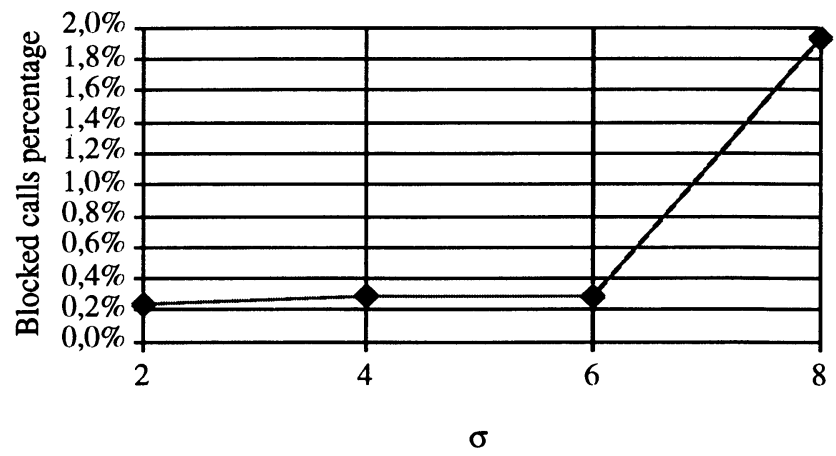

Figure 4. Blocked calls percentage vs. $\sigma$ with load $=1.0$.

As regards especially the average duration of connections, the values chosen are particularly short, in order to limit the length of the simulation runs necessary to obtain a significant number of events. However, one of the main purposes of the hierarchical scheme we have been using is that of coping, to a certain extent, with dynamic variations in the traffic characteristics; in our cases, with relatively short connections, this is achieved by keeping the reallocation interval also relatively short. In the case of longer connections (with the same traffic intensity), the situation would not be substantially different from this point of view.

In the following, the notation $(i, h)$ indicates node $i$ and class $h$. A Poisson distribution has been assumed for packet arrivals in all simulations.

\section{Behaviour with different $\sigma$}

In all the following simulations we have used a traffic pattern equally subdivided between isochronous and asynchronous. The isochronous traffic is the same for each class of every user station. The following data generates a total traffic flow which exploits the network potential to the utmost. We refer to this traffic flow as an offered load 1; an offered load " $x$ " corresponds to the same data except that $\lambda_{1}^{(i, h)}, \mathrm{i}=1,2,3,4, h=1,2,3$ and $\lambda_{2}$ are multiplied by $x$.

The network characteristics (corresponding to offered load 1) are:

$$
\begin{aligned}
& M=4 ; \quad H=3 ; \quad n^{(1)}=4, n^{(2)}=2, n^{(3)}=1 \\
& \lambda_{1}^{(i, 1)}=1.64 \quad \lambda_{1}^{(i, 2)}=3.28 \quad \lambda_{1}^{(i, 3)}=6.56 \quad \text { calls } / \mathrm{s} \forall \mathrm{i} \\
& 1 / \mu^{(i, h)}=4.5 \mathrm{~s} \forall i, \forall h \\
& \lambda_{2}=708,000 \text { packets } / \mathrm{s} ; \quad \mathrm{BWB}=8 \\
& \text { Propagation delay between adjacent nodes }=5 \mu \mathrm{s}
\end{aligned}
$$




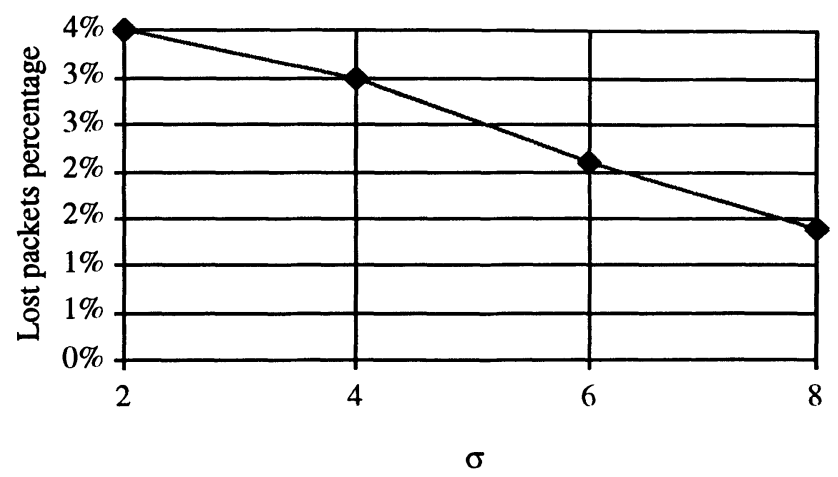

Figure 5. Lost packets percentage vs. $\sigma$ with load $=1.1$.

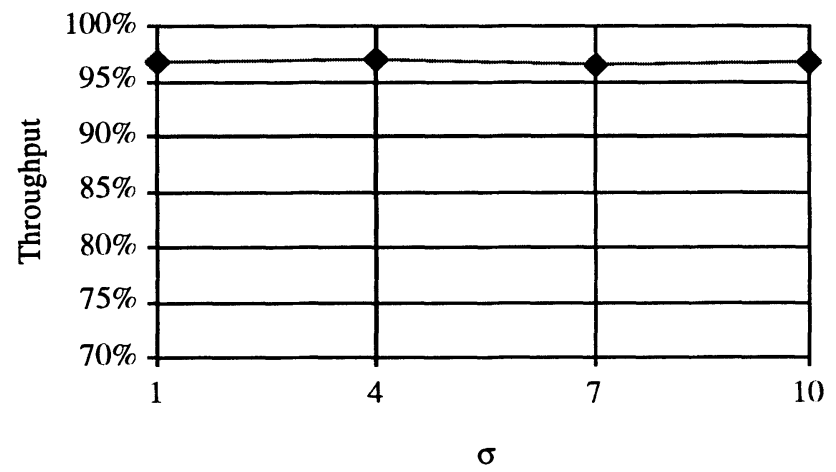

Figure 6. Throughput vs. $\sigma$ with load $=1.1$.

Figures 4, 5 and 6 represent the system behaviour with increasing values of $\sigma$ and a fixed offered load of 1.0. Moreover they show the blocked calls percentage, the lost packets percentage and the overall throughput of the network, respectively.

The effectiveness of $\sigma$ in controlling the share of the capacity between asynchronous and isochronous traffic without influencing the global performance becomes evident from the plots under examination. In fact, for increasing values of $\sigma$ the blocked calls percentages increase and lost packets percentages decrease, while the throughput does not change.

\section{Behaviour with increasing load}

The following plots show the behaviour of the network with increasing values of offered load. The characteristics of the network and the reference offered load value are the same as introduced in the previous situation. We have chosen $\sigma=6$, which appears to give the best 


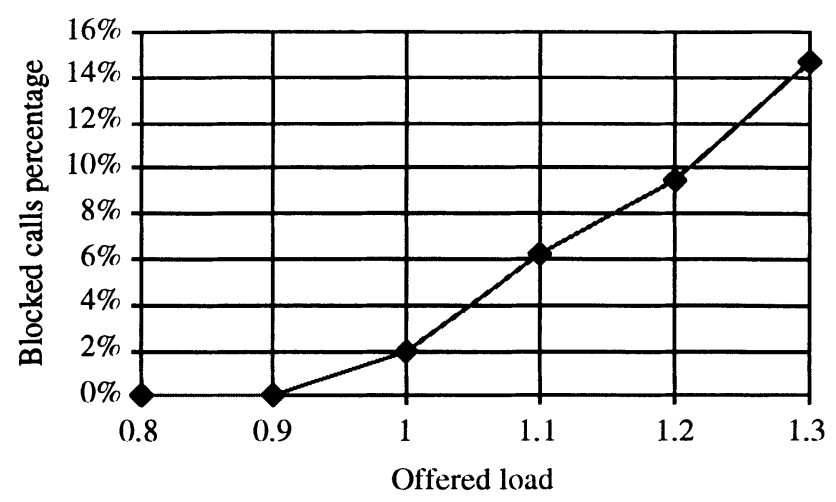

Figure 7. Blocked calls percentage with increasing load and $\sigma=6$.

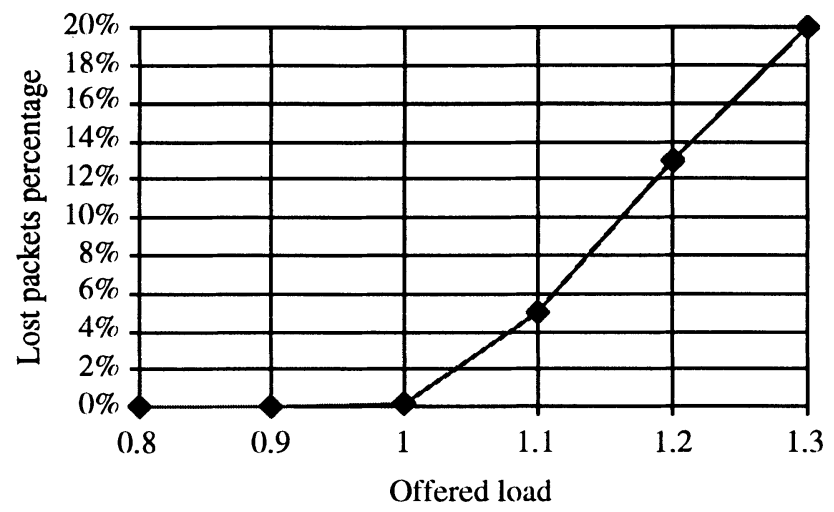

Figure 8. Lost packets percentage with increasing load and $\sigma=6$.

overall performaces.

Figures 7, 8 and 9 show the blocked calls percentage, the lost packets percentage and the overall throughput of the network, respectively. It can be seen that the percentage of refused calls is near zero up to an offered load of 0.9 and that the percentage of lost packets starts to become significant from an offered load of 1.1. Moreover, for saturation values of the offered traffic, the throughput behaviour (Figure 9), has values near the physically achievable maximum $(100 \%)$, which shows that the overall performance of the network is rather satisfactory.

In Figure 10, the percentage of blocked calls is represented for a value of the offered load that significantly exceeds the saturation value. The two plots refer to the overall loss per isochronous class (over all nodes) and per node (over all isochronous classes), respectively. The high losses (owing to the high load) are nevertheless shared quite fairly among the nodes; 


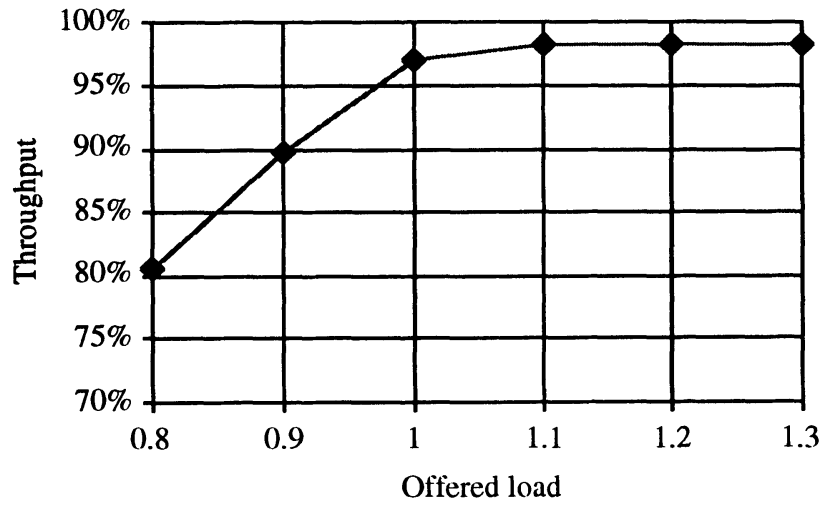

Figure 9. Throughput with increasing load and $\sigma=6$.
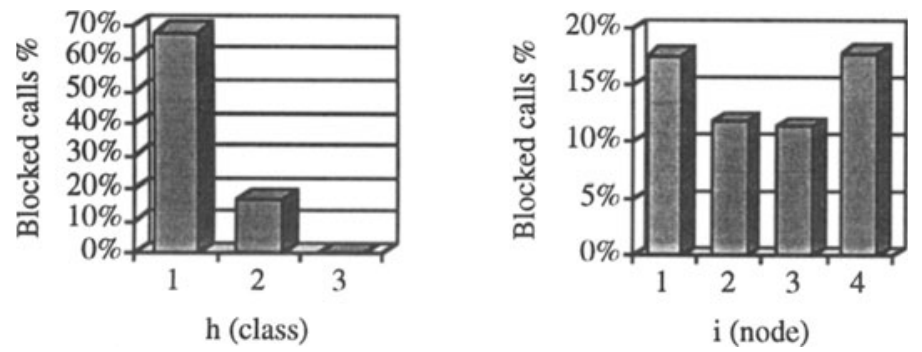

Figure 10. Blocked and generated calls with $l o a d=1.3$ and $\sigma=6$.

on the other hand, the unbalanced service among the different classes is mainly due to fact that the refusal of a call belonging to a high speed class leaves a larger number of slots available to other potential incoming calls than the refusal of low speed ones. Moreover, this behaviour can be altered at will, by means of different values of weighting coefficients assigned to the various classes in (13).

\section{CONCLUSIONS}

We have defined and analyzed a hierarchical management and control scheme for the integration of isochronous and asynchronous traffic flows in a MAN based on hybrid TDM frames. The analytical model takes into account the presence of multiple isochronous traffic classes, differentiated by user station and transmission speed, whereas packets are supposed 
to be handled by a distributed multiaccess protocol, whose details must not be necessarily known to the network manager. The isochronous traffic flows have been modeled as continuous time Markov chains, and the network manager optimization problem has been discretized over events.

The scheme has been tested by means of several simulation experiments, whose efficiency in terms of the computations involved results much greater than that of former discrete time formulations of the problem. The results show that the controlled network allows to sustain a high load, while keeping the QoS requirements; moreover, it is capable of achieving a balance between the isochronous and asynchronous traffic.

\section{REFERENCES}

Aicardi, M. Bolla, R. Davoli F and. Minciardi, R. (1993) A parametric optimization approach to admission control and bandwidth assignment in hybrid TDM networks. International Journal of Digital and Analog Communication Systems, 6, 15-27.

Bertsekas, D. and Gallager, R. (1987) Data Networks. Prentice-Hall, Englewood Cliffs, NJ.

Bolla, R. and Davoli, F. (1993) Dynamic hierarchical control of resource allocation in an integrated services broadband network. Computer Networks and ISDN Systems, 25, 10, 1079-87.

Bolla, R. Davoli, F. Maryni, P. Nobile, G. Pitzalis and G. Ricchebuono, A. (1994) Definition and evaluation of an adaptive performance management strategy for a hybrid TDM network with multiple isochronous traffic classes. In Network Management and Control (eds. I.Frisch, M.Malek and S.Panwar), Plenum Publ. Corp., New York, 2,. 487-501.

IEEE 802.6 (1990) Distributed Queue Dual Bus, Metropolitan Area Network, Draft Standard, Version D.15.13.

Hajek, B. (1984) Optimal control of two interacting service stations. IEEE Transactions on Automatic Control, AC-29, 491-99.

Kraimeche, B. and Schwartz, M. (1985) Analysis of traffic access control strategies in integrated service networks. IEEE Transactions on Communications, COM-33, 108593.

Maglaris, B.S. and Schwartz, M. (1982) Optimal fixed frame multiplexing in integrated lineand packet-switched communication networks. IEEE Transactions on Information Theory, IT-28, 273-83.

Malinowski, K. (1989) Hierarchical control under uncertainty: formal and practical aspects. Proceeding of 5th IFAC Symposium on Large Scale Systems, Berlin, Germany.

Rosberg, Z. Varaiya, P.P. Walrand, J.C. (1982) Optimal control of service in tandem queues. IEEE Transactions on Automatic Control, AC-27, 600-10.

Ross, K.W. and Chen, B. (1988) Optimal scheduling of interactive and non interactive traffic in telecommunication systems. IEEE Transactions on Automatic Control, 33, 261-7.

Ross, K.W. and Tsang, D.H.K. (1989) Optimal circuit access policies in an ISDN environment: a Markov decision approach. IEEE Transactions on Communications, COM-37, 934-9.

Serfozo, R.F. (1979) An equivalence between continuous and discrete time Markov Decision Processes. Operations Research, 27, 3, 616-20.

Sohraby, K. et al. , Eds .(1991) Special Issue on Congestion Control in High-Speed Packet 
Switched Networks. IEEE Journal on Select. Areas Communication, 9 , 7.

Viniotis, I. and Ephremides, A. (1987) Optimal switching of voice and data at a network node. Proceeding of 26th IEEE Conference on Decision and Control, Los Angeles, CA, 1504-07.

Wolff, R. (1987) Stochastic Modeling and the Theory of Queues. Prentice-Hall, Englewood Cliffs, NJ.

Zukerman, M. (1989-A) Bandwidth allocation for bursty isochronous traffic in a hybrid switching system. IEEE Transactions on Communications, COM-37, 1367-71.

Zukerman, M. (1989-B) Circuit allocation and overload control in a hybrid switching system. Computer Networks and ISDN Systems, 16, 281-98.

Raffaele Bolla was born in Savona, Italy, in 1963. He took the laurea degree in Electronic Engineering from the University of Genoa in 1989 and the Ph.D. degree in Telecommunications at the Department of Communications, Computer and Systems Science (DIST) of the University of Genoa, in 1994. He is currently teaching a course in Telecommunication Networks at DIST. His current research interests are in management and control of STM and ATM networks, multimedia communications and routing in high speed networks.

Franco Davoli was born in Genoa, Italy, in 1949. He received the "laurea" degree in Electronic Engineering from the University of Genoa in 1975. Since 1985 he has been Associate Professor and since 1990 Professor of Telecommunication Networks at the University of Genoa, where he is with the Department of Communications, Computer and Systems Science (DIST). From 1989 to 1991 and again since 1994, he has been also with the University of Parma, Italy, where he teaches a course in Telecommunication Networks.

His current research interests are in bandwidth allocation, admission control and routing in STM and ATM networks, multimedia communications and services, and integrated mobile radio networks. 